Pensamiento Crítico No. 7, pág. 71 - 83

\title{
El Significado del Etanol en el Mercado de los Combustibles
}

Pedro Barrientos Felipa

Resumen: El mundo de los biocombustibles, dentro del cual se encuentra el etanol, es nuevo, a pesar de que estos tienen miles de años siendo utilizados. Recién nos estamos dando cuenta de su importancia, pero también de los efectos adversos que se pueden generar. El artículo expone la perspectiva de uno de esos biocombustibles, el etanol, el cual puede tener una importante consecuencia en la economía de algunas regiones del Perú, pero también riesgos sociales que deben ser tomados en cuenta desde un inicio. Caso contrario puede estar germinando un nuevo problema.

Palabras claves: biocombustibles, etanol

\section{Presentación}

A inicios del presente siglo, se ha intensificado la preocupación por el medio ambiente, lo cual ha llevado a la búsqueda de diversas alternativas para mejorarlo. Una de esas alternativas se da a través del uso de nuevos combustibles, lo cual como toda actividad económica también debe ser rentable para los inversionistas. Es así como aparece, con mayor impulso, el etanol, combustible producido a partir de una diversidad de productos agrícolas y que hoy está causando diversas investigaciones para tener conocimiento de hasta donde son sus posibilidades de sustituir al petróleo o también si existe la suficiente demanda que ayude a madurar todo proyecto de inversión. 


\section{Pedro Barrientos Felipa}

Nuestro país, a través de inversionistas locales y extranjeros ha comenzado a analizar esta posibilidad. Incluso ya se tiene inversiones aseguradas para la producción de insumos (a partir de la caña de azúcar) del etanol. Existen otras alternativas de insumo, pero en nuestro caso se ha optado por el insumo citado, del cual ya se tiene experiencia, pero que se ha utilizado para la producción de azúcar. Esta nueva alternativa, en el mediano plazo puede ser una solución a la característica de ser un país importador de petróleo. Sin embargo, habría que ver quién será la demanda local o tal vez dirigir esta producción al mercado internacional. Si es local, dónde habrá mayor impulso, hacia el sector empresarial o al consumo masivo. Pero también hay productos sustitutorios como puede ser el gas natural.

\section{Los biocombustibles y el etanol}

La producción de biocombustibles es un tema que suena a nivel mundial; no se trata de un problema / solución de carácter regional o local. Es algo gravitante en muchos países del mundo. Así que nuestro país no esta ajeno a estas circunstancias. La menor producción de petróleo, además de los altos precios, está impactando fuertemente en la economía de muchos países, muy en especial de aquellos que son importadores netos de petróleo. Esto trae como consecuencia efectos en los diversos costos de la producción, desde los proveedores hasta el consumidor final. El agro no es ajeno a ninguna de estas consecuencias, y tiene una nueva posibilidad de participar en la producción de combustibles renovables, biocombustibles, y disminuir las emisiones tóxicas. La posibilidad de agregar un nuevo uso a las materias primas agrícolas ayuda a la expansión de la frontera de producción y representa un incentivo para el desarrollo de nuevas tecnologías que favorecen el incremento de la productividad y, consecuentemente, de la oferta de alimentos.

Tal como se puede prever, los biocombustibles, serían aquellos combustibles que tienen origen biológico, por lo cual el petróleo estaría incluido en tal concepto, considerando que el mismo procede de prehistóricos restos biológicos. Pero, existe el convencionalismo que trata a como biocombustible al combustible que tiene como origen biológico obtenido de manera renovable a partir del uso de restos orgánicos. En tal sentido, todo aquello que puede ser oxidado produce energía; si esta energía procede de plantas, entonces al ser quemada (oxidada) devuelve a la atmósfera el dióxido de carbono que la planta tomó del aire tiempo atrás. Por tanto, desde el punto de vista ecológico es un sistema que respeta el medio ambiente, pues no hay un aumento neto de gases de efecto invernadero.

Es posible utilizar este tipo de combustible como complemento o para aprovechar ciertos recursos que sería de todos modos desperdiciados. Aunque cubra un pequeño porcentaje de la producción de energía total aportaría su contribución de todos modos. Casi cualquier sustancia orgánica líquida o gasificable puede ser utilizada en un motor de explosión interna con la apropiada mecánica. Utilizar otros combustibles alternativos en nuestros actuales motores 


\section{El Significado del Etanol en el Mercado de los Combustibles}

sería más sencillo que la utilización de hidrógeno que se basa en una tecnología totalmente distinta.

Existe una oportunidad para saltos tecnológicos importantes una vez que los biocombustibles puedan establecerse como una alternativa económica del petróleo (1). Este desarrollo tecnológico no se restringirá a la transformación de la materia prima o a mejorar la tecnología en el consumo sino también invertir mas en la investigación agrícola para mejorar la productividad de la materia prima.

Existen tres importantes factores que promueven el desarrollo de los biocombustibles:

* Energía - sustituir combustibles basados en petróleo para aumentar la seguridad energética, disminuir la dependencia frente a la volatilidad de los precios de petróleo, bajar los costos de combustibles o de las importaciones, disminuir la dependencia de países políticamente inestables;

* Medio Ambiente - disminuir la emisión de Gases Invernaderos y de otros daños ambientales relacionados con la cadena del petróleo, como por ejemplo los derrames de petróleo;

* Desarrollo Rural / Agrícola - apoyar a la agricultura nacional, mejorar la situación económica de las áreas rurales y de los ingresos de los agricultores.

Grafico 1 - Los “drivers” de los biocombustibles

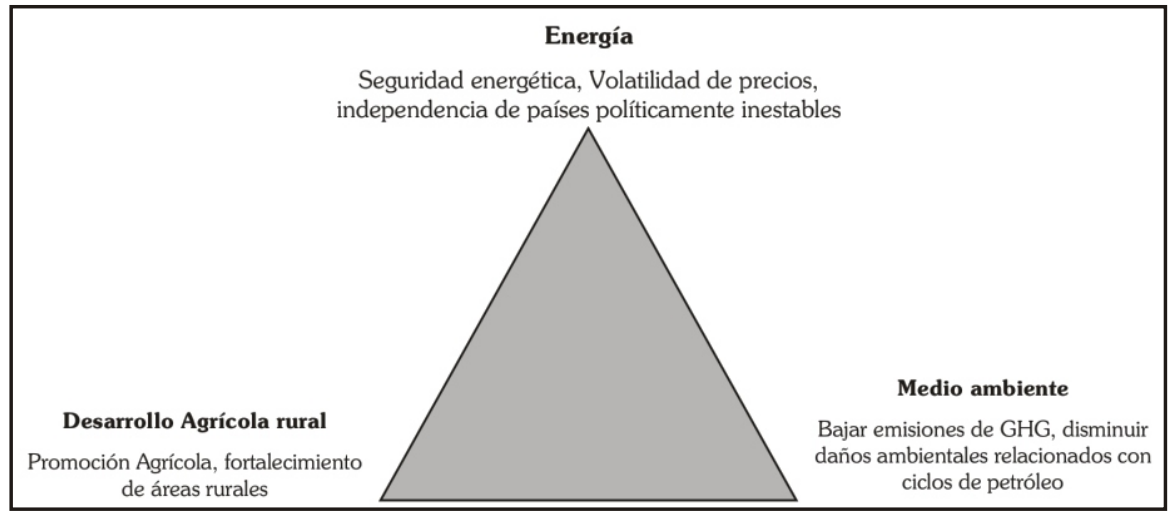

Fuente : Pfaumann, Peter

Sin embargo, en opinión de Bravo y Altieri (1), ambos sostienen que el cultivo masivo de maíz, caña de azúcar, soja, palma y otros cultivos utilizados como insumos y que son impulsados por la industria agroenergética no reducirán las emisiones de gases de efecto invernadero, pero si desplazará a miles de agricultores, disminuirá la seguridad alimentaria y acelerará la deforestación y la destrucción del medioambiente en el Sur Global. El avance de la "frontera 


\section{Pedro Barrientos Felipa}

agrícola" para la producción de biocombustibles es un atentado contra la soberanía alimentaria de las naciones en desarrollo, en tanto la tierra para producción de alimentos está crecientemente siendo destinada a alimentar los automóviles de los pueblos del Norte. La producción de biocombustibles también afecta directamente a los consumidores con un incremento en el costo de los alimentos. Debido al hecho de que más del $70 \%$ de los granos en EUA son utilizados como piensos, se puede esperar que al doblar o triplicar la producción de etanol suban los precios del maíz, y como consecuencia, el precio de la carne. La demanda de biocombustible en este país ha estado vinculada a un incremento masivo en el precio del maíz que condujo a un reciente aumento del $400 \%$ en el precio de la tortilla en México.

Tal como lo menciona la Organización de las Naciones Unidad en su informe "Energía sostenible. Un marco para la toma de decisiones" (Mayo, 2007) el rápido crecimiento de la bioenergía ofrece muchas oportunidades, pero también implica peligros y concesiones. "Es necesario estudiar detenidamente el impacto económico, social y medioambiental de la bioenergía antes de decidir cómo se quiere desarrollar de rápido y que tecnologías, políticas y estrategias de inversión seguir", advierte el informe.

El informe pone de relieve los numerosos beneficios de la bioenergía en relación con la mitigación de la pobreza, el acceso a los servicios energéticos, el desarrollo rural y las infraestructuras rurales. Analiza el impacto potencial de la bioenergía sobre la seguridad alimentaria, el cambio climático, la biodiversidad y los recursos naturales, el empleo y el comercio. Igualmente identifica los puntos clave que los responsables de la toma de decisiones necesitan considerar.

"A menos que se establezcan políticas para la protección de espacios amenazados, se garantice un uso socialmente aceptable de la tierra y se desarrolle la bioenergía de una forma sostenible, el daño social y medioambiental puede en algunos casos superar los beneficios", señala el documento. Es más, incluso los cultivos bioenergéticos "sostenibles" pueden tener un impacto negativo si sustituyen a los bosques primarios, lo que conlleva "la producción de elevadas cantidades de carbono procedente del suelo y la biomasa forestal, eliminando así cualquier beneficio de los biocombustibles durante décadas", añade el informe.

En todo caso, para darnos una idea del mundo de los biocombustibles, podemos recurrir a la propuesta de Food and Agriculture Organizatión (FAO), organismo de la Naciones Unidas, quien expone un sistema detallado de biocombustibles (3), el cual hace un reconocimiento del emplazamiento básico en el que se produce la biomasa. Así observamos si el biocombustibles está relacionado con la actividad forestal, agrícola o municipal. "La inclusión de un grupo sobre la utilización de agrocombustibles tiene por objeto distinguir los biocombustibles clásicos (que suelen estar relacionados con la explotación forestal) de los más modernos, orientados básicamente a la plantación anual o plurianual."

Continúa la Organización detallando que los grupos establecidos en relación con la oferta son subdivisiones importantes que identifican claramente el origen de los biocombustibles. En lo 


\section{El Significado del Etanol en el Mercado de los Combustibles}

que respecta al usuario final, se puede observar la diversidad de combustibles que se pueden producir para cada grupo, lo que permite comparar y verificar los datos relativos tanto a la oferta como a la demanda. En la ultima columna del cuadro 1 se citan los diferentes tipos de combustibles, primarios, secundarios e incluso terciarios, que se pueden utilizar para generar calor, electricidad y energía. Frecuentemente, los combustibles secundarios y terciarios proceden de la biomasa en bruto producida por varias fuentes tras la aplicación de unos procesos de transformación bastante complejos.

\section{Tabla No.1 : Clasificación de los biocombustibles}

\begin{tabular}{|c|c|c|}
\hline $\begin{array}{l}\text { En relación con la } \\
\text { producción, oferta }\end{array}$ & Grupos comunes & $\begin{array}{l}\text { En relación con los usuarios, } \\
\text { ejemplos de demanda }\end{array}$ \\
\hline $\begin{array}{l}\text { Combustibles de madera directos } \\
\text { Combustibles de madera } \\
\text { indirectos } \\
\text { Combustibles de madera } \\
\text { recuperados } \\
\text { Combustibles derivados de } \\
\text { madera }\end{array}$ & COMBUSTIBLES DE MADERA & $\begin{array}{l}\text { Sólidos: leña (madera en bruto, } \\
\text { astillas, aserrín, y pallets), carbón } \\
\text { vegetal } \\
\text { Líquidos: licor negro, metanol y } \\
\text { aceite pirolítico } \\
\text { Gases: productos procedentes de } \\
\text { la gasificación y la pirolisis, gases } \\
\text { de los combustibles mencionados } \\
\text { supra }\end{array}$ \\
\hline $\begin{array}{l}\text { Cultivos usados como } \\
\text { combustibles } \\
\text { Subproductos agrícolas } \\
\text { Subproductos de origen animal } \\
\text { Subproductos agroindustriales }\end{array}$ & AGROCOMBUSTIBLES & $\begin{array}{l}\text { Sólidos: paja, tallos, cáscaras, } \\
\text { bagazo y carbón vegetal de los } \\
\text { combustibles mencionados supra } \\
\text { Líquidos: etanol, aceite vegetal en } \\
\text { bruto, aceite "diester, metanol y } \\
\text { aceite pirolítico } \\
\text { Gases: biogás, productos } \\
\text { procedentes de la gasificación y la } \\
\text { pirolisis de agrocombustibles }\end{array}$ \\
\hline Subproductos de origen municipal & $\begin{array}{c}\text { SUBPRODUCTOS DE ORIGEN } \\
\text { MUNICIPAL }\end{array}$ & $\begin{array}{l}\text { Sólidos: residuos sólidos de origen } \\
\text { municipal (RSM) } \\
\text { Líquidos: fango de aguas } \\
\text { residuales, aceite pirolítico y de } \\
\text { RSM } \\
\text { Gases: gas procedente de } \\
\text { vertederos, gas procedente de } \\
\text { tratamiento de fangos cloacales. }\end{array}$ \\
\hline
\end{tabular}

Fuente : FAO - Guia para encuestas de demanda, oferta y abastecimiento de combustibles de madera.

Uno de esos biocombustibles es el etanol. En diversas partes del mundo se viene hablando últimamente acerca de la posibilidad que tiene el sector agrícola de incursionar en la producción de etanol, para su uso como alcohol carburante en motores de combustión interna. El principal atractivo de un programa de esta naturaleza estribaría en la reducción de las emisiones de $\mathrm{CO} 2$, comparativamente con los combustibles fósiles; contribuyéndose de paso al 


\section{Pedro Barrientos Felipa}

cumplimiento de los acuerdos de la Conferencia Internacional sobre Cambio Climático, (Protocolo de Kyoto), de la cual nuestro país es suscriptor. Sin embargo, cualquier iniciativa que pretenda seguirse en nuestro país al respecto, deberá necesariamente considerar otros efectos que van más allá de la producción de un combustible menos contaminante y más barato.

El Etanol es el alcohol etílico producido a partir de la fermentación de los azúcares que se encuentran en los productos vegetales (cereales, caña de azúcar, remolacha o biomasa). Dependiendo de su fuente de obtención, su producción implica fundamentalmente el proceso de separación de las azúcares, y la fermentación y destilación de las mismas.

En la actualidad el etanol es el biocombustible líquido de mayor aceptación. La posibilidad de usarse en forma exclusiva o en mezcla con otros combustibles fósiles y el hecho que se puede elaborar a partir de una amplia gama de cultivares, le otorgan ventajas para su difusión. Sin embargo, la producción 'comercial competitiva' del mismo afronta diversas restricciones según regiones y países. El costo y la seguridad para el suministro continuo de la materia prima seleccionada, son factores que determinan la posibilidad para su producción bajo condiciones de mercado. La caña de azúcar y el maíz son las principales especies utilizadas en el mundo para elaborar etanol.

Existen tres fuentes de materias primas para la producción del Etanol:

* En el primer grupo tenemos a los granos básicos: maíz, trigo, cebada, sorgo y papa. De estos productos, se consigue el almidón que a partir de un proceso de hidrólisis se convierta en azúcares.

* En el segundo grupo, tenemos a la caña de azúcar y la remolacha que son transformadas en melaza y luego se inicia el proceso para la obtención del Etanol.

* En un tercer grupo, se han clasificado a la madera, los desechos municipales y los desperdicios forestales, a partir de los cuales se obtienen las celulosas que siguen un proceso de hidrólisis para convertirse en azúcar.

En lo que corresponde a la producción mundial, Brasil encabeza la lista de los principales países productores; gran parte de su mercado automotriz ya utiliza biocombustibles; es un importante usuario de este combustible. Le siguen Estados Unidos e Indonesia. Los tres países acumulan el $64.0 \%$ de la producción mundial total. La producción mundial de etanol, tiene una tendencia creciente esto debido que en el mundo esta sucediendo un cambio energético en el cual los biocombustibles representan una gran alternativa de sustitución de los combustibles fósiles. Pese a lo cual no existe conformado, estructurado y debidamente consolidado un mercado de etanol en el mundo; pero que se va perfilando por el enorme interés que su empleo viene despertando en todo el ámbito internacional. El país predominante en el mercado de etanol siendo el primer productor y exportador mundial de etanol es Brasil que utiliza como materia prima la caña de azúcar, el segundo productor más grande de etanol a nivel mundial es EE.UU. que utilizan como materia prima al maíz. Hoy en día no hay otro elemento que pueda 


\section{El Significado del Etanol en el Mercado de los Combustibles}

reemplazar a la gasolina o al petróleo, como el etanol. Por esta razón la demanda de etanol como combustible ha aumentado por razones económicas, sociales y medioambientales.

Siete millones de automóviles en Brasil utilizan 100\% de etanol (5) y el resto de los 17 millones de automóviles $45 \%$. Si todos estos vehículos en lugar de consumir alcohol consumieran gasolina, Brasil dejaría de ahorrar en su balanza comercial más de tres mil millones de dólares por año, dos a cuatro millones de brasileños se quedarían sin trabajo. La atmósfera del planeta aumentaría cada año su contenido de dióxido de carbono (CO2), en poco más de 47 millones de toneladas métricas de carbono puro, lo que es equivalente a más de 12 millones de carbono puro por año. De no ser porque Brasil escogió, hace más de treinta años, el camino del alcohol motor como insumo bioenergético, estaríamos en estos días más cerca de la cada vez inevitable catástrofe ecológica, derivada del cambio climático, producida por la contaminación ambiental, sobre todo la generación de gases que se envían a la atmósfera "descontroladamente".

Tabla No. 2: Producción mundial de Etanol - 2002

\begin{tabular}{|l|r|r|r|r|}
\hline País / Área & Cantidad (1) & Valor (2) & \% (3) & \$ (4) \\
\hline Brasil & 33.835 .433 & 11.740 .232 & 25,72 & 0,344 \\
\hline Estados Unidos & 26.810 .473 & 10.428 .040 & 22,845 & 0,392 \\
\hline Indonesia & 21.565 .331 & 7.148 .024 & 15,66 & 0,328 \\
\hline Argentina & 14.770 .564 & 4.732 .664 & 10,368 & 0,32 \\
\hline Tailandia & 10.984 .465 & 3.647 .256 & 7,99 & 0,336 \\
\hline Australia & 10.128 .424 & 3.536 .440 & 7,747 & 0,352 \\
\hline China & 7.305 .563 & 3.256 .592 & 7,134 & 0,448 \\
\hline Pakistan & 1.806 .990 & 619.136 & 1,356 & 0,344 \\
\hline Francia & 394.812 & 446.440 & 0,978 & 1,128 \\
\hline India & 269.474 & 91.616 & 0,201 & 0,336 \\
\hline Total & 127.871 .529 & 45.646 .440 & 100 & 0.36 \\
\hline
\end{tabular}

Fuente: Jtrade City, en base a datos de la Japan Tariff Association (1) Litros; (2)Cifras Jtrade son suministradas en $¥$. Los datos aquí consignados han sido convertidos a US\$; empleando el tipo de cambio promedio anual para el año 2002 de $¥ 125=$ U\$ 1. (3)Participación en el total de importaciones. (4)Precio promedio del año convertido a dólares.

Con los niveles sin precedentes que están alcanzando los precios del petróleo crudo, crecen las perspectivas mundiales para el etanol usado como combustible. La producción del etanol (4) derivado de plantas feculentas y azucareras, tales como la caña de azúcar y algunos cereales, aumentó un 53 por ciento, desde 30 mil millones de litros en 2000 hasta alrededor de 


\section{Pedro Barrientos Felipa}

46 mil millones de litros en 2005. Se prevé que para 2010 el consumo mundial de etanol alcance los 54 mil millones de litros, correspondientes a alrededor de un 1 por ciento del consumo mundial de petróleo (que para 2010 superará, según las estimaciones del Consejo Mundial de la Energía [2005], los 5'151'000 millones de litros). El interés creciente que suscita el combustible etanol obedece no sólo a las preocupaciones por los altos precios del petróleo sino también a otros motivos importantes. Entre los cuales cabe mencionar los siguientes: la necesidad de diversificar las fuentes de energía, el deseo de muchos países de reducir el gas de efecto invernadero de conformidad con el Protocolo de Kyoto, y la necesidad de estabilizar los precios de los productos básicos y ajustar las subvenciones agrícolas a las disposiciones de la OMC. Gracias a los avances tecnológicos y a los incentivos normativos, la industria del combustible etanol ya no se limita a unos pocos países (Brasil, Japón y los Estados Unidos) sino que está cobrando importancia también en otras partes del mundo, sobre todo China, la India y Tailandia. $\mathrm{El}$ incremento de la utilización de etanol tiene el potencial de crear una demanda apreciable de productos agrícolas relacionados con la energía y de influir ulteriormente en los mercados de productos básicos.

\section{El etanol en el mercado peruano}

En el año 2003 se promulgó la Ley No. 28054, Ley de promoción del mercado de biocombustibles, cuyo objeto fue establecer el marco general para promover el desarrollo de los biocombustibles, donde dice que "Este mercado se debe basar en la libre competencia y el libre acceso de la actividad económica". El objetivo de la misma es diversificar el mercado de combustibles, fomentar el desarrollo agropecuario y agroindustrial, disminuir la contaminación ambiental y ofrecer un mercado alternativo en la lucha contra las drogas.

La Ley Nro. 28055, Ley de Promoción del Mercado de Biocombustibles, busca promover el desarrollo del mercado de los biocombustibles sobre la base de la libre competencia y el libre acceso a la actividad económica, para tal fin en su Segunda Disposición Transitoria estableció la creación de una Comisión Técnica. Dicha comisión está encargada de proponer y recomendar las normas y disposiciones complementarias para el cumplimiento de la ley, observando los siguientes lineamientos básicos:

a) Elaborar el cronograma y porcentajes de la aplicación y uso del etanol anhidro, como compenente para la oxigenación de las gasolinas, así como el uso de biodiesel en el combustible diesel.

b) Proponer un programa de sensibilización a los usuarios y a las instituciones públicas hacia el uso de etanol anhidro y biodiesel. Esta Comisión Técnica tiene como antecedente el Grupo Técnico de Biocombustibles, el cual fue creado por Decreto del Consejo Directivo N 024-01-CD/ CONAM; el mismo que en Octubre del 2002, al finalizar sus actividades, propuso la iniciativa legislativa, base para la presente ley. 


\section{El Significado del Etanol en el Mercado de los Combustibles}

Dentro de este ambiente legal, hay que considerar que "a diferencia de Brasil, que produce caña de azúcar - principal insumo para el etanol - seis meses al año, en las tierras peruanas la actividad se desarrolla los 365 días. Esa, sin embargo, no sería la única ventaja comparativa para que inversionistas privados se interesen en el Perú para el negocio del biocombustible, explica el presidente de la Asociación Peruana de Productores de Azúcar y Bi ocombustibles, Julio Morriberón. De acuerdo con los cálculos de ese gremio, Brasil obtiene 13 toneladas de azúcar de una hectárea de caña de azúcar, mientras que el Perú alcanza 25 toneladas; por lo que la competitividad en el país está marcada por la calidad y el volumen que se puede obtener con la caña "(6)". Es importante señalar que, en el Perú la mayor parte de la producción local de caña se destina a la elaboración de azúcar.

Entre 1998 y 2003 la producción de caña de azúcar en el Perú se incrementó a un ritmo de $5,8 \%$ por año, siendo producida totalmente en la costa, principalmente en la Libertad y Lambayeque. En la actualidad en el país solo existe la posibilidad de producir 50000 litros/día, debido que no existe la industria del etanol. Existen dos complejos industriales azucareros productores de Etanol (Cartavio y Quimpac) que tienen por principal destino la exportación, lo están realizando pero a menor escala. El Perú posee el mayor rendimiento a nivel mundial en la producción mundial de caña de azúcar, este un importante aliciente para producir etanol en base a la caña de azúcar, pese a ello no tenemos una elevada participación en la producción de caña, como otros países.

En la selva existe alrededor de 2 millones de hectáreas potencialmente disponibles para la producción de caña de azúcar y/ o sorgo dulce, que son utilizadas para cultivos con poca rentabilidad o que en algunos casos no son explotados por razones económicas.

Ante este panorama se desarrolló un proyecto para la elaboración de etanol en el valle del Huallaga. Dicho proyecto tiene como objetivo abastecer el mercado local, en un primer momento la selva; así como de exportar los excedentes de producción. En abril del 2003, Petroperú y el consorcio encargado del proyecto conformado por las empresas Coler \& Colantonio (EEUU), Naturel (EEUU) y Coimex (Brasil), firmaron un convenio mediante el cual la refinería de Iquitos compraría la producción de etanol del valle del Huallaga por diez a os, a partir de julio del 2004.

Para transportar el Etanol desde la Selva hasta la Costa, se construirá un alcoholducto desde el alle del Huallaga hasta el Puerto de Bayóvar (7), con una longitud aproximada de 1 029 ilómetros. La inversión calculada en dicho proyecto para los diez años es de US 185 millones.

Hay varias iniciativas en marcha para producir etanol. Maple-Etanol, con una inversión proyectada de US 100 millones, cultivará caña de azúcar en 11000 hectáreas en el proyecto Chira - Piura e instalará una planta productora de etanol, dando empleo directo a 1000 personas. Se prevé que en 2008 entregará su primer litro de etanol al mercado. Por otro lado, inversionistas españoles de Bio Terra han manifestado su interés en construir una planta de 


\section{Pedro Barrientos Felipa}

etanol en Lambayeque con una inversión de US 180 millones.

El proyecto de producción de biocombustibles del Grupo Romero también va tomando forma. Con la concesión de 3000 hectáreas en Piura y tierras adicionales en urimaguas, la empresa Agroenergía utilizará la caña de azúcar piurana y la palma aceitera de la Selva para abastecer a su red de estaciones; la producción se iniciará el 2007. También hay interés por parte de PETROBRAS y de inversionistas chinos para producir etanol en la zona norte del país.

\section{Tabla $N^{\circ}$ 3: Ventajas y desventajas del etanol}

\begin{tabular}{|c|c|}
\hline Ventajas & Desventajas \\
\hline $\begin{array}{l}\text { Se produce a partir de fuentes renovables } \\
\text { Presenta un elevado índice de octanaje (105), } \\
\text { favoreciendo la combustión y evitando el golpeteo. } \\
\text { Produce menos dióxido de carbono que la gasolina, } \\
\text { aunque el impacto total depende de los procesos de } \\
\text { destilación y la eficiencia de los cultivos } \\
\text { Genera menos monóxidos de carbono al utilizase como } \\
\text { aditivo en la gasolina. Con el uso de } 10 \% \text { de etanol en } \\
\text { la mezcla se puede lograr una reducción de } 25 \% \text { a } 30 \$ \\
\text { en las emisiones de CO. } \\
\text { Es menos inflamable que la gasolina y el diesel. } \\
\text { Baja toxicidad. } \\
\text { No emite compuestos de azufre. } \\
\text { La combinación de } 90 \% \text { de gasolina y } 10 \% \text { etanol } \\
\text { puede ser usado en los vehículos sin ninguna } \\
\text { modificación. }\end{array}$ & $\begin{array}{l}\text { Presenta menor poder calorífico que la gasolina, por lo } \\
\text { que requiere un mayor consumo. } \\
\text { Contiene } 2 / 3 \text { de la energía contenida en el mismo } \\
\text { volumen } \\
\text { La elaboración de etanol a partir de granos es más cara } \\
\text { que la gasolina. Aproximadamente } 1.5 \text { veces. } \\
\text { Presenta problemas de corrosión en partes mecánicas y } \\
\text { sellos. } \\
\text { En climas muy fríos presenta dificultades para el } \\
\text { encendido. } \\
\text { Genera emisiones de óxidos de nitrógeno y aldehídos } \\
\text { (contaminantes menores). } \\
\text { Para el uso de una mezcla de } 85 \% \text { etanol y } 15 \% \\
\text { gasolina se requiere de una adecuada modificación en } \\
\text { los motores. }\end{array}$ \\
\hline
\end{tabular}

Fuente : Ministerio de Industria, Comercio y Turismo "Perfil del etanol "

En febrero de este año, se presentó un estudio que plantea un análisis de la realidad del sector dedicado a la producción de alcohol (8), las tendencias del mercado, así como estrategias y políticas necesarias para desarrollar la producción de este recurso. El mencionado estudio se ejecutó gracias a la suscripción de un convenio de cooperación interinstitucional entre el Ministerio de la Producción, Ministerio de Agricultura, la Asociación Peruana de Productores de Caña de Azúcar y Biocombustibles, el Comité itivinícola de la Sociedad Nacional de Industrias, y TechnoServe Perú.

El Estudio de Competitividad del Etanol Anhidro, se realizó tomando en cuenta la próxima escasez de combustibles a nivel mundial, la sobreproducción y la dificultad de colocar 


\section{El Significado del Etanol en el Mercado de los Combustibles}

los excedentes de azúcar, el interés en reducir la contaminación ambiental y el aparente potencial competitivo que tiene el Perú para producir etanol anhidro. El informe explica que tenemos la ventaja del clima de las principales zonas de producción de caña de azúcar (insumo principal en la creación del etanol) debido a que todo el año es período de cultivo y cosecha, aspecto muy importante que le ha permitido ser actualmente el país con el mayor rendimiento de toneladas métricas de caña obtenidas por hectárea cosechada (114.03 Tm./ha), encontrándose muy por encima de los principales productores mundiales de etanol como Brasil y EE. UU. (72.87 y 72.52 $\mathrm{Tm}$./ha. respectivamente). Sin embargo, los costos de producir etanol en Perú (en los programas pilotos realizados) son muchos más altos que en Brasil, siendo en Perú 0.2286 y en Brasil 0.1321 por litro.

En opinión de Freddy Flores (Gerente General de la Asociación de Productores de Azúcar y Biocombustibles) opina que el Perú cuenta con condiciones excepcionales para la producción de Etanol, pero no es suficiente. En el mundo, se ha iniciado un acelerado desarrollo del etanol orientado a la expansión del mercado, sobre la base de políticas públicas promotoras para el ingreso de inversiones. La competitividad de estas inversiones se sustenta en la investigación y desarrollo tecnológico de la parte agrícola. En tal sentido, el Perú necesita de un Centro de Investigaciones en Caña de Azúcar para encarar con eficiencia el potencial de desarrollo del etanol (9)

\section{Tabla $N^{\circ}$ 4: Legislación promotora en otros países}

\begin{tabular}{|c|l|c|}
\hline País & \multicolumn{1}{|c|}{ Incentivos a la producción de biocombustibles } & Año \\
\hline Brasil & $\begin{array}{l}\text { PROALCOHOL } \\
\text { Estableció precios de etanol por debajo al de las gasolinas } \\
\text { Otorgó préstamos para incrementar su capacidad productiva } \\
\text { Redujo los impuestos para los vehículos que usan alcohol } \\
\text { Las estaciones de servicio fueron obligadas a vender alcohol } \\
\text { Contenido mínimo: 25\% de etanol en las gasolinas }\end{array}$ & 1979 \\
\hline Ecuador & $\begin{array}{l}\text { Exonera de Impuesto a la Renta y de aranceles a la maquinaria y equipos. } \\
\text { Exime de todos los impuestos en la constitución de empresas. Vigencia 10 } \\
\text { a 12 años) Ley No 2005 - 20 }\end{array}$ & 2005 \\
\hline Argentina & $\begin{array}{l}\text { Amortización a cuenta de ganancias y devolución anticipativa del IVA a las } \\
\text { inversiones para producir biocombustibles } \\
\text { Exime del "Impuesto a los combustibles líquidos y al gasoil" a la parte de } \\
\text { biocombustibles mezclada con derivados del petróleo. } \\
\text { Ley que promueve el Desarrollo Industrial de los biocombustibles }\end{array}$ & 2005 \\
\hline
\end{tabular}




\section{Pedro Barrientos Felipa}

\begin{tabular}{|c|l|c|}
\hline México & $\begin{array}{l}\text { Fomenta la construcción d infraestructura orientada a la producción de } \\
\text { biocombustibles } \\
\text { Crea mecanismos de financiamiento y promueve programas regionales } \\
\text { que incentiven la producción y uso de biocombustibles. } \\
\text { Ley para el Desarrollo y Promoción de los Bioenergéticos }\end{array}$ & 2006 \\
\hline Paraguay & $\begin{array}{l}\text { Incluye a los productores en los beneficios tributarios previstos en las } \\
\text { leyes №s 60/90 y 2421/04 que exoneran del pago de impuestos, dan un } \\
\text { tratamiento especial al Sector Agropecuario. Ley № 2748 }\end{array}$ & 2004 \\
\hline Colombia & $\begin{array}{l}\text { Declara el etanol exento del Impuesto al Valor Agregado } \\
\text { Exonera del “Impuesto global”. y de una sobretasa al alcohol } \\
\text { carburante que se mezcle con la gasolina. (Ley № 693, Ley № 788) }\end{array}$ & 2001 \\
\hline
\end{tabular}

Fuente : Perspectivas del etanol en el Perú.

Gráfico No. 3 - Perú :

Tres aspectos claves para invertir en etanol

MEDIDAS

Obligatoriedad del Uso Incremento gradual hasta $20 \%$

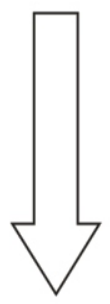

El uso opcional fomenta consumo de combustibles fósiles y aditivos importados contaminantes
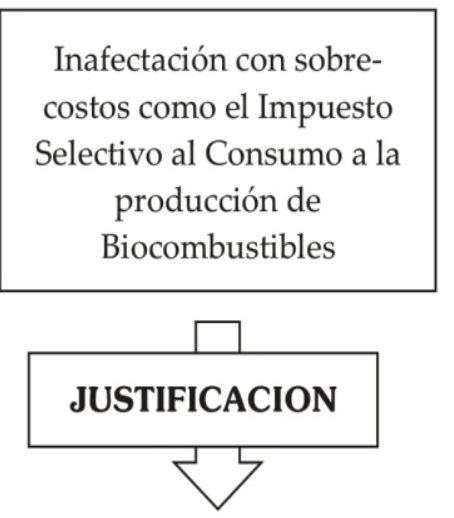

Aplicación de ISC resta competitividad y desalienta inversiones ( $\mathrm{p}$. e. GLP y GNV)
Precisión del Etanol como variante del alcohol etílico dentro de la Ley de Promoción Agraria

Fuente: Perspectivas del etanol en el Perú 


\section{El Significado del Etanol en el Mercado de los Combustibles}

\section{A modo de conclusión}

El etanol es un importante biocombustible que tendrá un efecto significativo en nuestra economía: integrará nuevas áreas de cultivo, generará nuevos puestos de trabajo, incentivará a los inversionistas. Pero también tiene sus riesgos, los cuales deben ser evaluados. Es cierto que es menos contaminante para la comunidad, es más barato que los combustibles fósiles como el petróleo, pero el costo social también deberá ser incluido.

\section{Bibliografía}

1. Pfaumann, Meter "Biocombustibles La formula mágica para las economías rurales "Bano Interamericano de Desarrollo. Noviembre 2006

2. Elizabeth Bravo, Elizabet y Altieri; Miguel "La tragedia social y ecológica de la producción de biocombustibles agrícolas en América"

3. Food and Agriculture Organization "Guía para encuestas de demanda, oferta y abastecimiento de combustibles de madera" 2002

4. Food and Agriculture Organization "El aumento de precios del petróleo crudo estimula la demanda de productos agrícolas relacionados con el etanol". Junio 2006

5. Rivas, Luis "Experiencia en el desarrollo de etanol en el mundo". Seminario Internacional de Fuentes alternativas de energía. ADE . Agosto 2006

6. El Comercio / Economía/Pág. B-2 / 08-Febrero-2007

7. Ministerio de Industria, Comercio exterior y Turismo "Perfil de mercado de etanol"

8. Asociación de productores de Caña de azúcar y biocombustibles. "Estudio de competitividad del etanol anhídrido" Febrero 2007

9. Flores Herrera, Freddy "Perspectivas del etanol en el Perú" Asociación Peruana de Productores de Caña de Azúcar y Biocombustibles. Mayo, 2006 